\title{
Serum KL-6 differentiates neuroendocrine cell hyperplasia of infancy from the inborn errors of surfactant metabolism
}

\author{
M L Doan, ${ }^{1}$ Elidemir, ${ }^{1}$ M K Dishop, ${ }^{2}$ H Zhang, ${ }^{1}$ E 0 Smith, ${ }^{3}$ P G Black, ${ }^{4}$ R R Deterding, ${ }^{5}$ \\ D M Roberts, ${ }^{6}$ O A Al-Salmi, ${ }^{7}$ L L Fan ${ }^{1}$
}

${ }^{1}$ Pediatric Pulmonary Section, Department of Pediatrics, Baylor College of Medicine, Texas Children's Hospital, Houston, Texas, USA; ${ }^{2}$ Department of Pathology, Baylor College of Medicine, Texas Children's Hospital, Houston, Texas, USA;

${ }^{3}$ Department of Pediatrics,

Baylor College of Medicine,

Houston, Texas, USA

${ }^{4}$ Department of Pediatrics, University of Missouri at Kansas City, Children's Mercy Hospital, Kansas City, Missouri, USA;

${ }^{5}$ Department of Pediatrics, University of Colorado Health Science Center, Children's Hospital, Denver, Colorado, USA; ${ }^{6}$ Pediatric Breathing Disorders Clinic, Anchorage, Alaska, USA; Department of Child Health, Royal Hospital, Oman

Correspondence to: Dr L L Fan, Texas Children's Hospital, 6621 Fannin, CC1040.00 Houston, TX 77030, USA; Ilfan@texaschildrens.org

Received 13 September 2008 Accepted 2 February 2009

Published Online First

22 February 2009

\section{ABSTRACT}

Background: The study was conducted in order to determine if the glycoprotein $\mathrm{KL}-6$ is a useful biomarker in differentiating neuroendocrine cell hyperplasia of infancy (NEHI), a benign form of children's interstitial lung disease, from the more severe inborn errors of surfactant metabolism (IESM), since their clinical presentation can be similar.

Methods: Serum KL-6 levels were measured in 10 healthy control children, 6 with NEHI and 13 with IESM (4 with surfactant protein $\mathrm{C}$ (SP-C) and 9 with $\mathrm{ABCA} 3$ mutations). The initial clinical presentation, findings on previous CT scans and interstitial lung disease (ILD) scores at the time of KL-6 testing were compared. Correlations of KL-6 levels with age and with interval from lung biopsy were evaluated.

Results: The median (range) KL-6 levels were 265 (1-409), 194 (47-352), 1149 (593-4407) and 3068 (726-9912) U/ml for the control, NEHI, SP-C and ABCA3 groups, respectively. When compared with the control and NEHI groups, median KL-6 levels were significantly higher in the SP-C $(p<0.01 ; p=0.01$, respectively) and $A B C A 3$ groups $(p<0.001 ; p=0.001$, respectively): however, there was no difference between the control and NEHI groups $(p=0.91)$. An inverse relationship was seen between KL-6 levels and age in the IESM groups, but not in the NEHI or control groups. Children with NEHI had similar presenting clinical features and were equally symptomatic at the time of KL-6 measurement as those with IESM.

Conclusions: Children with NEHI have normal KL-6 levels, in contrast to those with IESM, who have elevated serum KL-6 levels; serum KL-6 may be a useful biomarker in distinguishing between these entities when their clinical presentations overlap.

Children's interstitial lung disease (chILD) encompasses a heterogeneous group of rare diffuse lung disorders with varying prognoses. More severe forms include the inborn errors of surfactant metabolism (IESM), such as the mutations in the surfactant protein $\mathrm{C}$ (SP-C) and $\mathrm{ABCA} 3$ genes. ${ }^{12}$ In contrast, neuroendocrine cell hyperplasia of infancy (NEHI) is considered a benign form, with no reported mortality. ${ }^{3}$ Although the underlying pathology of these conditions is very different, their clinical presentations may overlap.

In addition to severe respiratory failure in the newborn period, ${ }^{2}{ }^{5}$ SP-C or ABCA3 mutations can also present later in infancy with persistent retractions, tachypnoea, hypoxaemia, crackles and poor growth. ${ }^{6-12}$ The family history can be negative, even in patients with autosomal dominant SP-C mutations. ${ }^{68}$ Findings on high-resolution CT (HRCT) of the chest in infants with SP-C or $\mathrm{ABCA} 3$ mutations typically show bilateral diffuse ground-glass opacification with septal thickening. ${ }^{70} 1213$ Mutational analyses for the SP$\mathrm{C}$ and $\mathrm{ABCA} 3$ genes are now available through commercial reference laboratories, but are occasionally non-diagnostic, even when the histopathology is consistent with IESM. ${ }^{214}$ The histological findings of the lung disease due to proven SP-C and ABCA3 mutations consistently include hyperplasia of type 2 pneumocytes and interstitial inflammation. ${ }^{2}$

Children with NEHI also present from the neonatal period to late infancy with features identical to those described for IESM. Chest radiographs demonstrate hyperinflation and increased interstitial markings, ${ }^{3}$ and HRCT typically shows patchy ground-glass opacification distributed centrally and in the right middle lobe and lingula, as well as diffuse air trapping. ${ }^{4}$ While these CT findings can be distinctive, the significant clinical manifestations seen with NEHI can raise concerns for more severe chILD, and a diagnostic open lung biopsy is frequently undertaken in these children. The histology in NEHI, however, is notable for the absence of significant interstitial inflammation and is instead characterised by increased numbers of pulmonary neuroendocrine cells within the airway epithelium and lobular parenchyma. $^{3}$

Despite their similar clinical presentation, the marked differences between the histology of IESM and NEHI raise the question of whether noninvasive biomarkers may be able to differentiate severe from benign forms of chILD or be able to predict the presence or absence of significant interstitial inflammation. One such potential biomarker is KL-6, a glycoprotein that is preferentially expressed in the normal lung by type 2 alveolar cells and bronchiolar epithelial cells. ${ }^{15}$ Investigators have demonstrated increased expression of KL-6 on regenerating alveolar cells in patients with various types of ILD, with correspondingly high serum levels. ${ }^{16}{ }^{17}$ Such elevated KL6 levels have also been reported in various forms of chILD, ${ }^{18}$ while normal levels are seen in asthma, bronchiolitis, pertussis, common viral and bacterial pneumonias, and acute eosinophilic pneumonia. ${ }^{19-2}$ Furthermore, while KL-6 levels have been correlated with disease activity, they do not appear to be affected by steroid therapy. ${ }^{22}$ 
We sought to characterise serum KL-6 levels in children with SP-C and ABCA3 mutations and in those with NEHI. Since alveolar epithelial cell hyperplasia is a common histological feature of IESM, but not of NEHI, we hypothesised that serum KL-6 would be elevated in IESM, but not in NEHI.

\section{METHODS}

\section{Subjects}

A blood sample was collected from 10 healthy children, 6 with NEHI and 13 with IESM (4 with SP-C and 9 with ABCA3 mutations). Study subjects included all children with diagnosed NEHI or IESM who are followed or have been evaluated at our institution (5 NEHI, 11 IESM), as well as subjects recruited via collaborating physicians (1 NEHI, 2 IESM).

The diagnosis of NEHI was established by surgical lung biopsy, with all biopsies reviewed by the paediatric lung pathologists at Texas Children's Hospital, using guidelines of $>75 \%$ of airways containing neuroendocrine cells, $>10 \%$ neuroendocrine cells in any individual airway and the presence of prominent neuroepithelial bodies. The children with IESM were diagnosed by mutational analysis under a research protocol at the Johns Hopkins University School of Medicine or by the CLIA (Clinical Laboratory Improvement Amendments)-certified Johns Hopkins DNA Diagnostic Laboratory. The control group consisted of 10 healthy children who were recruited from families whose parents were members of the Pediatric Pulmonary Section at Texas Children's Hospital. Exclusion criteria in the control group included a history of febrile illness or upper respiratory tract infection during the 3 weeks prior to enrolment or a history of pneumonia during the 3 months prior to enrolment.

The records of the children with NEHI or IESM were reviewed to collect information on the initial clinical presentation. Available CT scans from the first 18 months of life were reviewed for the pattern of ground-glass opacification and the presence of septal thickening. As children with NEHI are known to improve with age, ${ }^{3}$ and the clinical course of those with IESM can be variable, ${ }^{12}$ the clinical status of each subject at the time of KL- 6 testing was assessed using the ILD severity score ${ }^{23}$ in order to relate to serum KL-6 levels. Levels were also correlated with age and with time interval from lung biopsy for each group.

The control subjects and eight of the children with ABCA3 mutations have previously been reported. ${ }^{12}{ }^{18}$ Results of the current study have also been presented in abstract form. ${ }^{24}$ The Institutional Review Board of the Baylor College of Medicine approved this study, and informed written consent was obtained from each subject's parent or legal guardian.

\section{Serum KL-6 measurements}

A $3 \mathrm{ml}$ venous blood sample was obtained from each subject for analysis of KL-6. Samples were drawn in a plain container, processed for serum and stored at $-70^{\circ} \mathrm{C}$ until used. Each serum sample was then analysed in duplicate for KL- 6 using commercially available ELISA kits and following the protocol of the manufacturer (Sanko Junyaku, Tokyo, Japan). The reported value for each test was the average of the duplicate results. The detection range of the kit for standard $\mathrm{KL}-6$ is $1-20 \mathrm{U} / \mathrm{ml}$. The within-run and between-run coefficients of variation were no more than $10 \%$, and the recovery rate was $90-108 \%$.

\section{Statistical analysis}

Group comparisons were done using non-parametric procedures. The Kruskal-Wallis test was used for comparing $\geqslant 3$ groups, followed by the Mann-Whitney test for pairwise comparisons. The relationshipa between KL-6 levels and age at testing and interval from lung biopsy were analysed using the Spearman correlation in order to avoid normality assumptions.

\section{RESULTS}

\section{Clinical presentation}

The 6 children with NEHI were all born at term. Their ages at symptom onset ranged from birth to 5 months. All presented with tachypnoea and hypoxaemia, while retractions, persistent crackles and poor growth were less frequent features (table 1). Most of the patients had at least one hospitalisation (one child had >20) due to respiratory illness, but none had suffered respiratory failure. Chest CT obtained at 3-9 months of age consistently revealed bilateral ground-glass opacification, usually with a patchy distribution that was limited to the right middle lobe, lingula and central regions. In one child, however, a repeat study 4 weeks after his initial one showed a change from the typical patchy distribution to that of diffuse panlobar involvement of ground-glass opacification (fig 1A,). Septal thickening was absent in all studies. Open lung biopsy was undertaken in these children at ages 5-17 months, and the histology consistently demonstrated an increased proportion of airways containing bombesin-immunopositive cells (79-100\%), an increased proportion of immunopositive cells in immunopositive airways (12-32\%) and the presence of neuroepithelial bodies, but very little if any inflammation and no hyperplasia of type 2 alveolar cells. Half of these children had received chronic systemic steroid therapy at some point during infancy (but none in the year prior to KL- 6 testing), and the mean KL-6 levels for the treated and untreated children were not significantly different $(229 \mathrm{U} / \mathrm{ml}$ vs $190 \mathrm{U} / \mathrm{ml}$, respectively, $\mathrm{p}=0.7)$. All 6 children were still alive at ages 4 months to 8 years but continued to have hypoxaemia, either continuously or only with sleep, at the time of KL-6 testing.

Ten of the children with SP-C or ABCA3 mutations had symptom onset in infancy. Two had respiratory failure in the newborn period (one recovered and one underwent lung transplantation). Three others (one with SP-C and two with ABCA3 mutations) developed respiratory failure beyond the newborn period but within the first 3 months of life, after having presented with tachypnoea, retractions and hypoxaemia of only several weeks duration; two of them subsequently died. The remaining five patients had similar clinical manifestations but did not have rapidly progressive deterioration (table 1). Chest CT scan was performed by age 18 months (range 318 months) in six of these children. Four studies were available

Table 1 Clinical features of subjects who presented in infancy

\begin{tabular}{llr}
\hline & NEHI & IESM \\
\hline Tachypnoea & $6 / 6$ & $10 / 10$ \\
Retractions & $5 / 6$ & $7 / 10$ \\
Crackles & $4 / 6$ & $5 / 10$ \\
Hypoxaemia & $6 / 6$ & $8 / 10$ \\
Poor growth & $2 / 6$ & $7 / 10$ \\
Respiratory failure & $0 / 6$ & $5 / 10$ \\
CT* findings $^{*}$ Diffuse GGO & & \\
$\quad$ Patchy GGO & $1 / 6$ & $4 / 6$ \\
Septal thickening & $6 / 6$ & $2 / 6$ \\
\hline
\end{tabular}

*Available studies from the first 18 months of life.

GGO, ground-glass opacification; IESM, inborn errors of surfactant metabolism; NEHI neuroendocrine cell hyperplasia of infancy. 
Figure 1 CT scans of the chest in infants with neuroendocrine cell hyperplasia of infancy (NEHI), ABCA3 mutations and surfactant protein $C$ (SP-C) mutation. ( $A$ and $B$ ) Images at different time points in the same patient with biopsy-proven NEHI, demonstrating classic $(A)$ and more diffuse $(B)$ patterns of ground-glass opacification. (C) CT in a 3-month-old infant with $A B C A 3$ mutations showing diffuse ground-glass opacification and very subtle septal thickening. (D) CT in another 3-month-old infant with $A B C A 3$ mutations revealing more patchy ground-glass opacification but with prominent interstitial thickening.
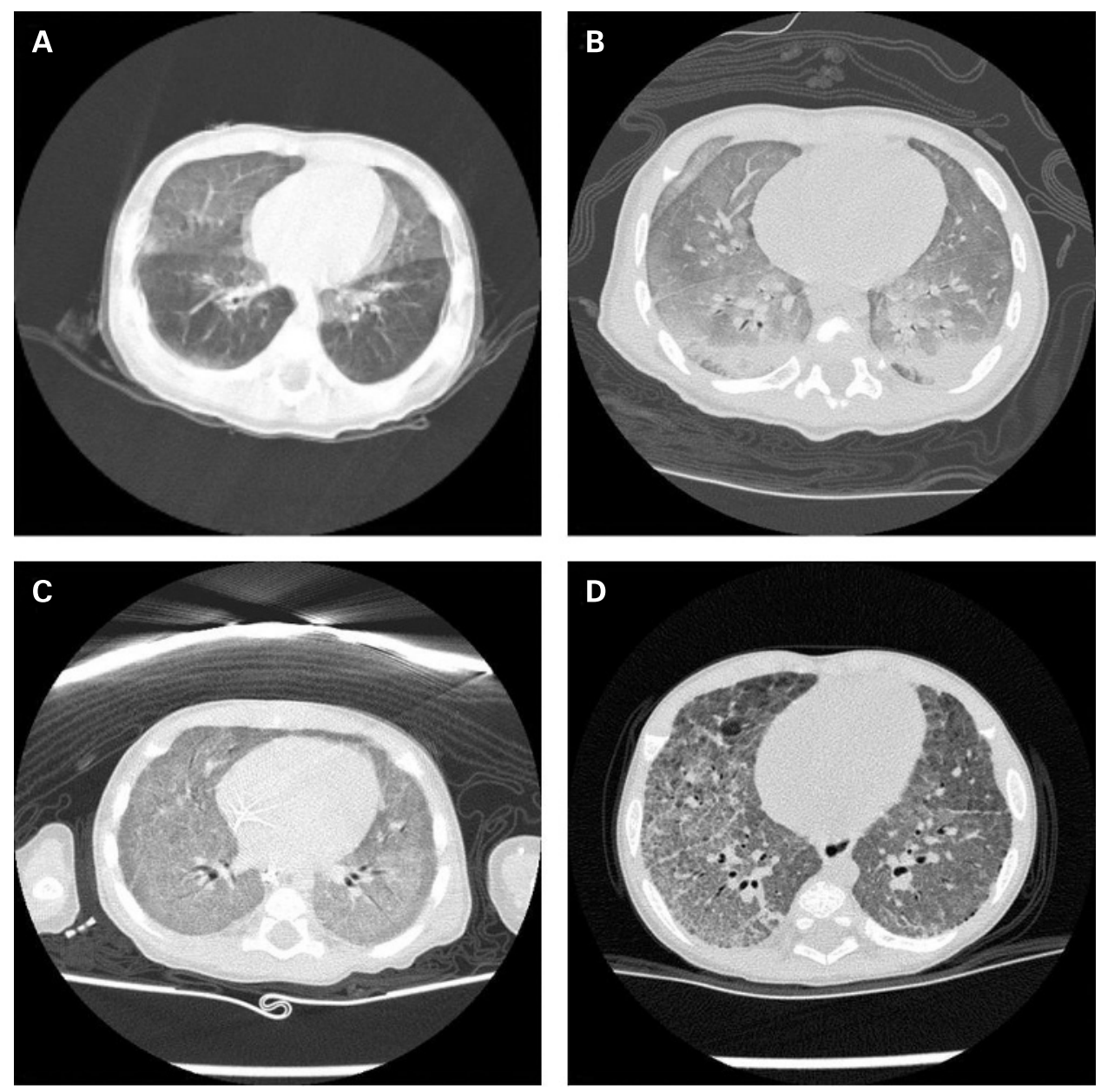

for review, while only the report was available in the remaining two. The majority showed diffuse ground-glass opacification, but multifocal, patchy involvement was also seen. Septal thickening was noted in 4/6 studies (fig 1C,D). For 12 of the enrolled children with SP-C or ABCA3 mutations, open lung biopsy was undertaken between ages 1 month and 7 years, and histological findings consistently included interstitial inflammation with type 2 alveolar cell hyperplasia.

\section{Serum KL-6 levels}

The median (range) serum KL-6 levels were 265 (1-409), 194 (47-352), 1149 (593-4407) and 3068 (726-9912) U/ml for the control, NEHI, SP-C and ABCA3 groups, respectively (fig 2). When compared with the control group, serum KL-6 levels were significantly higher in the SP-C $(p<0.01)$ and ABCA3 $(p<0.001)$ groups. Likewise, when compared with the NEHI group, KL-6 levels were also significantly higher in the SP-C $(p=0.01)$ and ABCA3 ( $p=0.001$ ) groups. If the SP-C and ABCA3 groups are combined and compared with the NEHI group, the differences are still highly significant $(p=0.001)$. On the other hand, there was no difference between the median values for the control and NEHI groups $(p=0.91)$. All serum KL-6 values for subjects with IESM were above $500 \mathrm{U} / \mathrm{ml}$, while all values for controls and patients with NEHI were below this level.

The median (IOR) age at the time of KL-6 testing was 11 years (9-12 years) for the healthy control children, 2.1 years (1.2-3.8 years) for the children with NEHI, 9.7 years $(0.8-15.4$ years) for those with SP-C mutations and 8.0 years (0.3-10.5 years) for those with ABCA3 mutations. When serum KL-6 levels were related to age and to the interval since lung biopsy, there were no correlations seen in the NEHI group $(R=0.26$, $p=0.62$ for both comparisons) or the control group $(R=-0.12$, $\mathrm{p}=0.74$ for comparison with age). On the other hand, there were significant inverse correlations demonstrated when serum KL-6 levels were related to age and to interval from lung biopsy in the patients with IESM (SP-C and ABCA3 combined) $(\mathrm{R}=-0.65, \mathrm{p}<0.05 ; \mathrm{R}=-0.69, \mathrm{p}<0.05$; respectively). In three children with ABCA3 mutations, serial follow-up KL-6 levels were also available and showed decline with increasing age (fig 3).

When comparing the clinical status of patients in each group in terms of requirement for supplemental oxygen at the time of KL- 6 testing using the interstitial lung disease (ILD) score, the median (range) score was 3.5 (3-4), 3.5 (1-4) and 4.0 (1-4) for the neuroendocrine cell hyperplasia of infancy (NEHI), surfactant protein $\mathrm{C}$ (SP-C) and ABCA3 groups, respectively $(p=0.86)$. In the NEHI group, the mean KL-6 level for the 3 children with an ILD score of 3 was $273 \mathrm{U} / \mathrm{ml}$, while the mean level for those with a score of 4 was $145 \mathrm{U} / \mathrm{ml}$.

\section{DISCUSSION}

Our report is the first to describe the differences between serum KL-6 levels in children with NEHI as compared with those with IESM. While these levels are expected to be normal in diseases predominantly involving the airways and elevated in ILD, our results suggest that serum KL- 6 may be able to help differentiate NEHI from the IESM in patients whose clinical presentation can be consistent with either condition. 


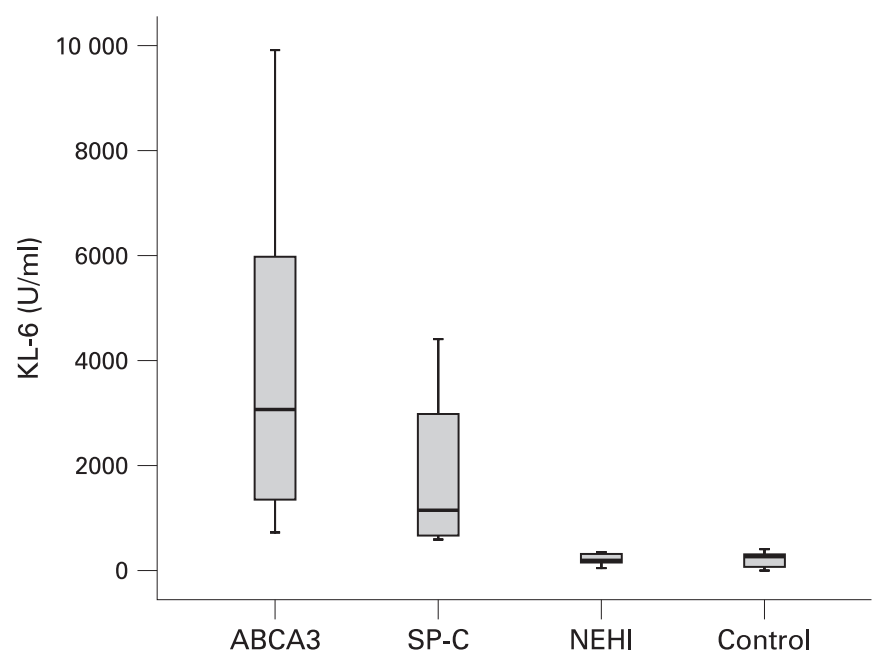

Figure 2 Serum KL-6 levels in the study groups (with group medians). $\mathrm{NEHI}$, neuroendocrine cell hyperplasia of infancy; SP-C, surfactant protein C.

Our findings have reaffirmed that the manifestations of NEHI can overlap with those of more severe chILD, such as the IESM. While none of the children with NEHI developed respiratory failure, this manifestation would not be a helpful early differentiating feature. Consistent with previous reports, ${ }^{34}$ we saw on the CT scans of all six children with NEHI a characteristic distribution of the ground-glass opacification and an absence of septal thickening. However, some of the children in our study with IESM who had CT scans in the first 18 months of life also had patchy involvement of ground-glass opacification, and some did not have septal thickening. Additionally, when one child in our study who was eventually diagnosed with NEHI underwent a repeat CT scan due to clinical deterioration, more diffuse ground-glass opacification was seen, prompting a diagnostic open lung biopsy. All six children with NEHI (only two of whom were primarily cared for at our institution) had been deemed to have lung disease significant enough to warrant an open lung biopsy by 27 months of age. The biopsies all showed the diagnostic features of NEHI and, despite the significant clinical manifestations, there was a lack of notable inflammation.

Our study also reiterates the need to consider early open lung biopsy in some cases of suspected IESM. Two of the children with ABCA3 mutations who presented beyond the neonatal period were symptomatic only for a few weeks before they developed rapidly progressive respiratory failure, leading to death. While mutational analysis allows for non-invasive diagnosis of the IESM, they routinely require 3-5 weeks for completion. CT findings of diffuse ground-glass opacification would suggest the presence of an IESM, especially if septal thickening is also seen, but these findings may not be consistently found. If lung transplantation is to be considered for treatment of an IESM, a prompt lung biopsy may be necessary in children with rapid progression of symptoms, as it may provide a presumptive diagnosis based on typical histological patterns and electron micrographic findings of abnormalities of lamellar bodies. ${ }^{5225}$ Furthermore, previous reports indicate that there are children with histological features consistent with an IESM in whom no mutations are found. ${ }^{2}{ }^{14}$ Thus, non-diagnostic genetic testing for IESM is not necessarily reassuring, and further evaluation with lung biopsy may still be required.
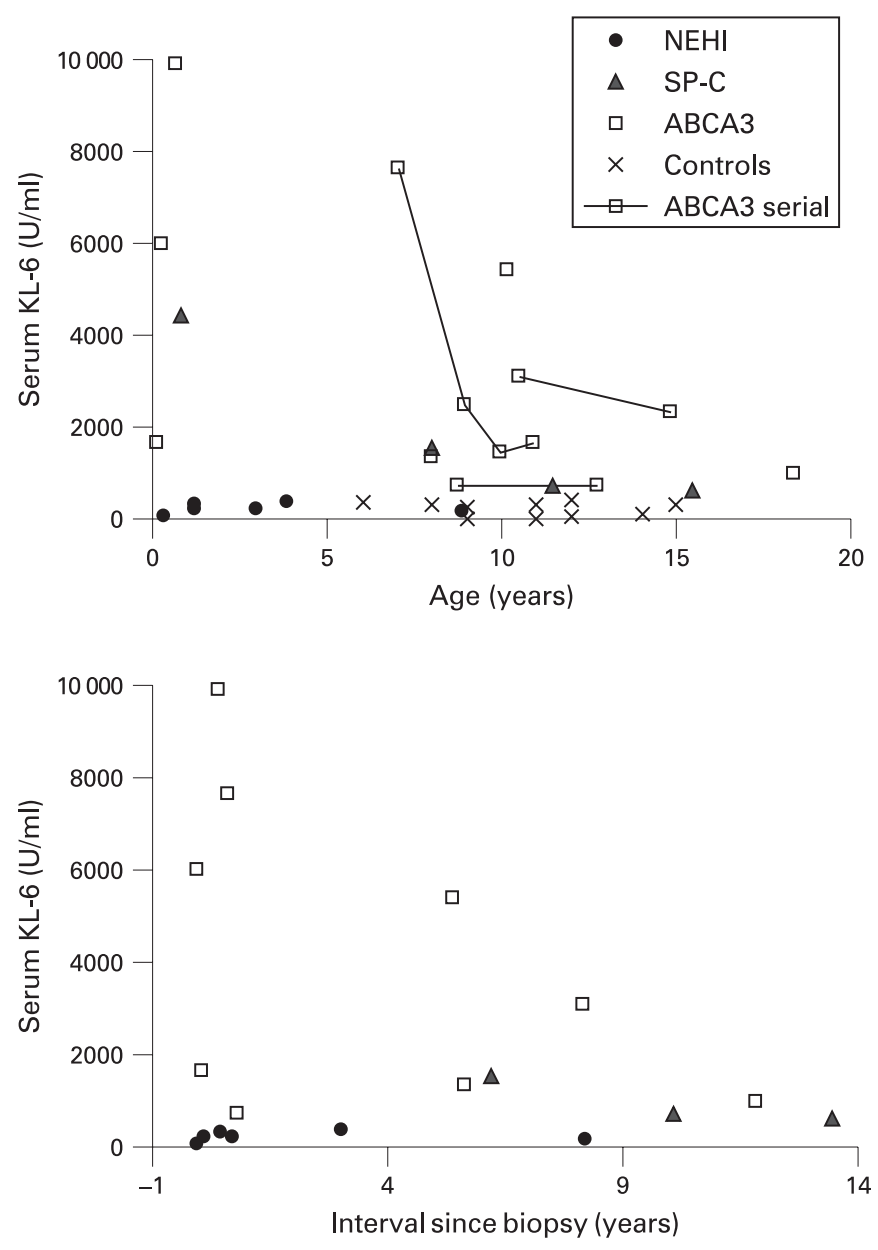

Figure 3 Serum KL-6 levels related to age (top) and interval since lung biopsy (bottom).

Because KL-6 is strongly expressed on the apical membrane of regenerating type 2 pneumocytes, ${ }^{16}$ and increased leak of KL-6 into the circulation seems to result from cell injury and/or inflammation-induced increased permeability of the alveolarcapillary membrane, ${ }^{26-28}$ measurement of serum KL-6 levels may be an ideal non-invasive method of assessing for the presence of interstitial inflammation. Elevated KL-6 levels in bronchoalveolar lavage (BAL) fluid have been demonstrated in pulmonary alveolar proteinosis, hypersensitivity pneumonitis, sarcoidosis, acute respiratory distress syndrome and idiopathic pulmonary fibrosis, ${ }^{22} 2930$ and in various ILDs elevated serum KL-6 levels have been correlated with BAL levels. ${ }^{26}{ }^{27}$ In adults, $500 \mathrm{U} / \mathrm{ml}$ has been suggested as an upper limit of normal for serum levels. ${ }^{21}$ While not as well defined in children, studies reporting healthy paediatric controls have consistently reported serum KL-6 levels below this cut-off and that these levels do not vary with age. ${ }^{18} 2028$ 31-34 In our study, there was no overlap in serum KL-6 levels between healthy children or children with NEHI and those with IESM, and a limit of $500 \mathrm{U} / \mathrm{ml}$ effectively separated the two former groups from the latter. Therefore, it would appear that a normal serum KL-6 level correlates with the absence of significant interstitial inflammation.

There are some apparent weaknesses of our study which need to be addressed. First, we recognise that the number of patients in each group is small. The rarity of these conditions makes it difficult to enrol significantly larger numbers of subjects within a reasonable amount of time, even within our referral 
population. However, the differences in serum KL-6 levels amongst the groups were so marked, without any overlap in individual values, that clinical and statistical significance was still demonstrated. Secondly, the mean age of the NEHI group was lower than that of the SP-C and ABCA3 groups, raising the possibility that differences in age and disease duration may have contributed to the differences in KL-6 levels. However, in the patients with SP-C or ABCA3 mutations, there was actually an inverse relationship between age and serum KL-6 levels. On the other hand, KL-6 levels did not vary with age in the children with NEHI, as was noted in our and other previously reported normal controls. ${ }^{19} 20$ Therefore, the differences in levels were most marked in the infants. Lastly, the cross-sectional design of the study resulted in measurement of KL-6 levels at various intervals after lung biopsy had been undertaken in many of the subjects and various treatments had been given. Thus, it is difficult to prove that a priori the serum KL-6 levels would have been helpful in the decision to proceed to a lung biopsy. However, there was no correlation between KL-6 levels and the interval between biopsy and KL- 6 measurement in the NEHI group, and all of these children were still symptomatic with some degree of hypoxaemia at the time of testing. On the other hand, an inverse relationship was again seen for this comparison in the patients with IESM. In the study, there was one young infant with NEHI and one with ABCA3 mutations who actually had serum KL-6 measured prior to undergoing open lung biopsy, and the levels were markedly different (47 U/ml vs $5978 \mathrm{U} / \mathrm{ml}$, respectively). Additionally, in three children with ABCA3 mutations, we were able to follow levels serially over a span of 4 years, and all showed a decline over time. Therefore, it does not appear that the differences in the serum KL-6 levels between children with NEHI and those with IESM can be explained solely because they were measured at various times after lung biopsy had been undertaken.

In conclusion, based on our findings, we think that serum KL-6 levels could be helpful in a diagnostic algorithm for the evaluation of an infant with suspected ILD. In the patient whose clinical course has not included respiratory failure and who has the characteristic distribution of ground-glass opacification limited to the right middle lobe, lingular and central regions, a normal serum KL-6 level would add confidence to the diagnosis of NEHI and may obviate the need for a diagnostic surgical lung biopsy. On the other hand, an elevated serum KL-6 level would raise concerns that another process involving significant interstitial inflammation or alveolar epithelial cell injury is more likely to be present. In this case, evaluation for an IESM should be undertaken by genetic testing, or by a lung biopsy if genetic testing is non-diagnostic or if rapidly progressive symptoms develop. Further studies are needed to evaluate serum KL-6 levels in other forms of mild and severe chILD, especially those also not associated with significant interstitial inflammation, in order to understand better its clinical utility in practice.

Acknowledgements: The authors would like to thank Drs Jason Fullmer, Rebecca Buckley, Kristen Meckelburg, Kenneth Van Wieren, Elsa Li and Mark Wilson for their assistance in obtaining samples, and the patients and families for their willingness to aid us with this research.

\section{Competing interests: None.}

Ethics approval: This study was approved by the Institutional Review Board of Baylor College of Medicine.

\section{REFERENCES}

1. Nogee LM, Dunbar AE, Wert SE, et al. A mutation in the surfactant protein $\mathrm{C}$ gene associated with familial interstitial lung disease. N Engl J Med 2001;344:573-9.
2. Shulenin S, Nogee LM, Annilo T, et al. ABCA3 gene mutations in newborns with fatal surfactant deficiency. N Engl J Med 2004;350:1296-303.

3. Deterding RR, Pye C, Fan LL, et al. Persistent tachypnea of infancy is associated with neuroendocrine cell hyperplasia. Pediatr Pulmonol 2005;40:157-65.

4. Brody AS, Crotty EJ. Neuroendocrine cell hyperplasia of infancy (NEHI). Pediatr Radiol 2006;36:1328.

5. Hamvas A. Inherited surfactant protein-B deficiency and surfactant protein-C associated disease: clinical features and evaluation. Semin Perinatol 2006;30:316-26.

6. Brasch $\mathbf{F}$, Griese $\mathbf{M}$, Tredano $\mathbf{M}$, et al. Interstitial lung disease in a baby with a de novo mutation in the SFTPC gene. Eur Respir J 2004;24:30-9.

7. Tredano $\mathbf{M}$, Griese $\mathrm{M}$, Brasch $\mathrm{F}$, et al. Mutation of SFTPC in infantile pulmonary alveolar proteinosis with or without fibrosing lung disease. Am J Med Genet 2004;126A:18-26.

8. Hamvas A, Nogee LM, White FV, et al. Progressive lung disease and surfactant dysfunction with a deletion in surfactant protein $\mathrm{C}$ gene. Am J Respir Cell Mol Biol 2004:30:771-6.

9. Chibbar R, Shih F, Baga M, et al. Nonspecific interstitial pneumonia and usual interstitial pneumonia with mutation in surfactant protein $\mathrm{C}$ in familial pulmonary fibrosis. Mod Pathol 2004;17:973-80.

10. Soraisham AS, Tierney AJ, Amin HJ. Neonatal respiratory failure associated with mutation in the surfactant protein C gene. J Perinatol 2006;26:67-70.

11. Saugstad OD, Hansen TWR, Ronnestad A, et al. Novel mutations in the gene encoding ATP binding cassette protein member A3 (ABCA3) resulting in fatal neonatal lung disease. Acta Paediatr 2007:96:185-90.

12. Doan ML, Guillerman RP, Dishop MK, et al. Clinical, radiological and pathological features of ABCA3 mutations in children. Thorax 2008;63:366-73.

13. Prestridge A, Wooldridge J, Deutsch G, et al. Persistent tachypnea and hypoxia in a 3-month-old term infant. J Pediatr 2006;149:702-6.

14. Bullard JE, Wert SE, Whitsett JA, et al. ABCA3 mutations associated with pediatric interstitial lung disease. Am J Respir Crit Care Med 2005;172:1026-31.

15. Kohno N, Akiyama M, Kyoizumi S, et al. Detection of soluble tumor-associated antigens in sera and effusion using novel monoclonal antibodies, KL-3 and KL-6, against lung adenocarcinoma. Jpn J Clin Oncol 1988;18:203-16.

16. Kohno N, Kyoizumi S, Awaya $Y$, et al. New serum indicator of interstitial pneumonia activity, sialyated carbohydrate antigen KL-6. Chest 1989;96:68-73.

17. Kobayashi J, Kitamura S. KL-6: a serum marker for interstitial pneumonia. Chest 1995;108:311-

18. Al-Salmi 0A, Walter JN, Colasurdo GN, et al. Serum KL-6 and surfactant proteins A and $D$ in pediatric interstitial lung disease. Chest 2005:127:403-7.

19. Imai T, Takase M, Takeda S, et al. Serum KL-6 levels in pediatric patients: reference values for children and levels in pneumonia, asthma, and measles patients. Pediatr Pulmonol 2002;33:135-41

20. Kubota M, Haruta T. The role of serum KL-6 measurement in common pediatric respiratory infections. J Infect Chemother 2006;12:22-4.

21. Daimon T, Tajima S, Oshikawa K, et al. KL-6 and surfactant proteins A and D in serum and bronchoalveolar lavage fluid in patients with acute eosinophilic pneumonia. Intern Med 2005:44:811-7.

22. Takahashi T, Munakata M, Suzuki I, et al. Serum and bronchoalveolar fluid KL-6 levels in patients with pulmonary alveolar proteinosis. Am J Respir Crit Care Med 1998;158:1294-8.

23. Fan LL, Kozinetz CA. Factors influencing survival in children with chronic interstitial lung disease. Am J Respir Crit Care Med 1997;156:939-42.

24. Doan ML, Zhang H, Elidemir 0, et al. Serum KL-6 levels in children with inborn errors of surfactant metabolism and neuroendocrine cell hyperplasia of infancy [abstract]. Chest 2006;130:156S

25. Edwards V, Cutz E, Viero S, et al. Ultrastructure of lamellar bodies in congenital surfactant deficiency. Ultrastruct Pathol 2005;29:503-9.

26. Kohno N, Awaya Y, Oyama T, et al. KL-6, a mucin-like glycoprotein, in bronchoalveolar lavage fluid from patients with interstitial lung disease. Am Rev Respir Dis 1993;148:637-42.

27. Inoue $\mathbf{Y}$, Barker $E$, Daniloff $E$, et al. Pulmonary epithelial cell injury and alveolarcapillary permeability in berylliosis. Am J Respir Crit Care Med 1997:156:109-15.

28. Imai T, Takase M, Takeda S, et al. Serum KL-6 levels in pediatric patients: reference values for children and levels in pneumonia, asthma, and measles patients. Pediatr Pulmonol 2002;33:135-41.

29. Kunitake R, Kuwano K, Yoshida K, et al. KL-6, surfactant protein A and D in bronchoalveolar lavage fluid from patients with pulmonary sarcoidosis. Respiration 2001;68:488-95.

30. Ishizaka A, Matsuda T, Albertine KH, et al. Elevation of KL-6, a lung epithelial cell marker, in plasma and epithelial lining fluid in acute respiratory distress syndrome. Am J Physiol Lung Cell Mol Physiol 2004;286:L1088-94.

31. Suzuki R, Suzuki $Y$, Itoh $K$, et al. A study of reference intervals for a serum KL-6 in normal children without respiratory disease and a serum marker for interstitial pneumonia. J Jpn Pediatr Soc 2001;105:703-10.

32. Kurotobi S, Maekawa S, Hara T, et al. Serum KL-6 level in newborns with meconium aspiration syndrome. Pediatr Int 2003;45:517-21.

33. Ogihara T, Hirano K, Morinobu T, et al. KL-6, a mucinous glycoprotein, as an indicator of chronic lung disease of the newborn. J Pediatr 2000;137:280-2.

34. Yamane M, Yokoyama. Serum KL-6 level and pulmonary function in preterm infants with chronic lung disease. Kobe J Med Sci 2004;50:131-40. 